\title{
Responsabilidad Social: Cumplimiento de directrices ISO 26000 frente a los requerimientos legales de seguridad y salud en el trabajo
}

\section{Social responsibility: Compliance with the ISO 26000 guidelines against the legal requirements of health and safety at work}

Luis Fernando Freire-Constante

Universidad Internacional del Ecuador, Quito, Ecuador

Ifreire@systemsvt.com

(iD https://orcid.org/0000-0002-9936-3041

Edison Daniel Yandún-Burbano

Universidad Rey Juan Carlos, Madrid, España

danielyandun@hotmail.com

(D) https://orcid.org/0000-0001-7871-1417

Erick Alberto Castro-García

Profesional Independiente, Quito, Ecuador

erick_al_cg@hotmail.com

(i) https://orcid.org/0000-0002-1916-2865

Sebastián Ricardo Castro-García

Secretaría de Educación Superior, Ciencia, Tecnología e Innovación, Senescyt, Quito, Ecuador srcastro@senescyt.gob.ec

iD https://orcid.org/0000-0002-3393-979X

Recepción: 16/11/2021 | Aceptación: 16/12/2021 | Publicación: 10/01/2022

Cómo citar (APA, séptima edición):

Freire-Constante, L.F., Yandún-Burbano, E.D., Castro-García, E.A., y Castro-García, S.R. (2022). Responsabilidad Social: Cumplimiento de directrices ISO 26000 frente a los requerimientos legales de seguridad y salud en el trabajo. INNOVA Research Journal, 7(1), 96-

108. https://doi.org/10.33890/innova.v7.n1.2022.1989

\section{Resumen}

La presente investigación es un estudio descriptivo no experimental de corte transversal, cuyo propósito es evidenciar el cumplimiento de Responsabilidad Social y prevención de riesgos 
Responsabilidad Social: Cumplimiento de directrices ISO 26000 frente a los requerimientos legales de seguridad y salud en el trabajo

laborales a través de las directrices de la Guía ISO 26000 para identificar los estándares de implementación en empresas ecuatorianas. Se utilizó la encuesta como instrumento de recolección de datos en los meses de marzo y abril del año 2021, contando con una participación de 388 profesionales y al ser un estudio de variables cualitativas se analizó las frecuencias y porcentajes de cumplimiento de las directrices del numeral 6.4.6 Asunto 4 sobre prácticas laborales: salud y seguridad ocupacional de la norma ISO 26000 Guía de Responsabilidad Social. El nivel de cumplimiento de las variables analizadas en gestión documental y buenas prácticas universales como entrega de equipos de protección personal, colectiva y exámenes ocupacionales son aceptables. La gestión en equidad y protección a personas vulnerables, el generar nuevas políticas que garantice la no persecución a empleados por evidenciar acciones y condiciones inseguras en sus lugares de trabajo y la falta de compromiso en la no participación y toma de decisiones de los organismos paritarios es menos frecuente en su cumplimiento. Se observa debilidades en la consecución de las directrices emitidas por la Guía de Responsabilidad Social en el contexto del compromiso, protección y equidad de todos los grupos de interés de las organizaciones. Debe ser reformulado el compromiso de gestionar integralmente los riesgos laborales ya que es una de las aristas principales de la Responsabilidad Social empresarial.

Palabras claves: prevención de accidentes; responsabilidad social; seguridad en el trabajo.

\begin{abstract}
This research is a descriptive non-experimental cross-sectional study, whose purpose is to demonstrate compliance with Social Responsibility and occupational risk prevention through the guidelines of the ISO 26000 Guide to identify the implementation standards in Ecuadorian companies. The survey was used as a data collection instrument in the months of March and April 2021, with the participation of 388 professionals and, being a study of qualitative variables, the frequencies and percentages of compliance with the guidelines of numeral 6.4.6.6 Subject 4 on labor practices: occupational health and safety of the ISO 26000 Guide to Social Responsibility were analyzed. The level of compliance with the variables analyzed in document management and universal good practices such as the provision of personal and collective protection equipment and occupational examinations are acceptable. The management in equity and protection of vulnerable people, the generation of new policies that guarantee the non-prosecution of employees for evidencing unsafe actions and conditions in their workplaces, and the lack of commitment in the non-participation and decision making of the joint bodies are less frequently complied with. Weaknesses are observed in the achievement of the guidelines issued by the Social Responsibility Guide in the context of commitment, protection and equity of all stakeholders of the organizations. The commitment to comprehensive management of occupational risks should be reformulated, as it is one of the main aspects of corporate social responsibility.
\end{abstract}

Key words: accident prevention; social responsibility; occupational safety.

\title{
Introducción
}

El numeral 6.4.6 de la ISO 26000, asunto 4 sobre prácticas laborales: salud y seguridad ocupacional, Guía de Responsabilidad Social, facilita las directrices de cumplimiento de gestión para las empresas y en concordancia con el Acuerdo Ministerial Nro. MDT-2018-135 que es el cuerpo legal vigente de estricto cumplimiento de la legislación ecuatoriana, estas dos herramientas de gestión son objeto del presente estudio para poder evidenciar el grado de cumplimiento y compromiso en lo referente a la prevención de riesgos laborales. 
La Responsabilidad Social como una parte de la estrategia empresarial busca responder las inconsistencias que ocurren entre los objetivos de rentabilidad y los objetivos sociales (Martinez Ferrero et al., 2013) se concentra en ir más allá del cumplimiento legal o la ley que se establece en un país, es decir, excede los estándares mínimos de conducta determinados en la normativa, sumado al principio de hacer lo correcto. Este último enfoque promueve la participación equitativa de todas las partes interesadas en la implementación de los elementos que contiene la Responsabilidad Social dentro de la organización (Olanipekun et al., 2021).

La filosofía actual para incorporar la sostenibilidad en los negocios es la perspectiva del triple resultado o Triple Bottom Line que se precisa como bienestar económico, calidad medioambiental y justicia social. Por tal razón se considera que muchos directores están descubriendo esta tríada de conceptos incluidos bajo esta representación, tratando de asignar recursos a múltiples cuestiones económicas, ambientales y sociales que incrementan el valor agregado del producto o servicio ofrecido (Gallardo et al., 2013).

El contemplar la Responsabilidad Social como parte de la estrategia corporativa de las empresas pone como ostensible la importancia que esta sea conocida por todos los grupos de interés (Escamilla Solano et al., 2016). Por esta razón la Organización Internacional de Normalización (ISO) promueve la Responsabilidad Social mediante la norma ISO 26000 y busca actualmente alinearse con los Objetivos de Desarrollo Sostenible (ODS) de las Naciones Unidas (Chandan y Das, 2017).

La norma de Responsabilidad Social ISO 26000 alienta a las empresas a ir más allá del cumplimiento de la normativa legal y contribuir al desarrollo sostenible. Otorga información acerca de los principios, su implementación, la identificación y la participación de las partes interesadas en aspectos fundamentales como los derechos humanos, la gobernanza, las prácticas laborales, el ambiente, las prácticas operativas justas, los consumidores y la participación activa en la comunidad (Creixans-Tenas et al., 2019), suministra un marco para la aplicación de la Responsabilidad Social, así como una breve revisión de métodos y directrices creíbles para la evaluación del impacto social (Pishvaee et al., 2021).

ISO 26000 facilita guías voluntarias, en lugar de requisitos, por lo que no puede ser certificada, a diferencia de otras normas ISO muy conocidas en el ámbito empresarial. Esta norma determina que la Responsabilidad Social, traduce principios en acciones efectivas, y colabora con prácticas integrales, se enfoca a todo tipo de organizaciones, independientemente de su actividad, tamaño o ubicación. La norma fue publicada en el 2010 tras cinco años de negociaciones entre partes interesadas a nivel mundial. En su creación participaron representantes de gobiernos, organizaciones no gubernamentales ONG, consumidores y representantes laborales de todo el mundo, lo que simboliza un consenso internacional (Chandan y Das, 2017).

Esta norma intenta ayudar a las organizaciones a abordar sus compromisos y proporcionar orientación práctica relacionada con la puesta en marcha de la Responsabilidad Social, la identificación y su relación con las partes interesadas y la credibilidad de los informes y afirmaciones realizadas (Castka y Balzarova, 2008). 
Responsabilidad Social: Cumplimiento de directrices ISO 26000 frente a los requerimientos legales de seguridad y salud en el trabajo

Las directrices del documento establecen siete materias fundamentales en su guía para la implementación de la Responsabilidad Social Empresarial como derechos humanos, prácticas laborales, gobernabilidad, medio ambiente, prácticas justas de operación, asuntos de consumidores, participación y desarrollo de la comunidad (Pulido, 2018), otorga una particular importancia al capital humano que labora en la empresa, pues son ellos la base de la evolución empresarial y del posicionamiento en el mercado (López Salazar et al., 2017).

Salud y seguridad en el trabajo en el Ecuador

El objetivo de la salud y la seguridad en el trabajo es prevenir y minimizar accidentes y enfermedades de carácter laborales mediante procesos sistemáticos que identifiquen, evalúen y controlen la gestión de riesgos en una organización (Liu et al., 2021) (Rajendran et al., 2021). Los estados de salud de las personas y su relación con el medio ambiente laboral es clave para el bienestar integral de los colaboradores (Romero Saldaña et al., 2019).

Las organizaciones deben promover la toma de conciencia e importancia de esta disciplina y deben ir más allá del cumplimiento de la normativa vigente en un país, esto se puede lograr a través de la implementación de sistemas de gestión voluntarios que aborden la cadena de valor integral garantizando la protección a todo su grupo de interés (López Gobernado et al., 2018) (Céspedes Socarrás y Martínez Cumbrera, 2016). Como principio base las organizaciones deben velar por la integridad y derechos de sus trabajadores más allá de una cancelación de salarios por prestación de sus servicios. El bienestar y la seguridad de los trabajadores debería estar como prioridad en toda actividad laboral (Shanmugasundar et al., 2021).

Algunas empresas abordan e implementan temas de Responsabilidad Social e incluyen programas enfocados a la gestión integral de riesgos (Sharma y Mishra, 2021), la importancia de proteger la salud de los trabajadores, avalar espacios de trabajo seguros, reducir riesgos, promover protocolos de vigilancia de la salud e implementar programas de prevención, soportan de alguna manera la dimensión social (Moreno y Villena, 2020), en este sentido las organizaciones buscan equilibrar lo social, ambiental y económico para cumplir con la sostenibilidad (Larrosa Moles y Martínez-Jarreta, 2020).

Dentro de la Responsabilidad Social, la Salud y Seguridad en el Trabajo es una parte fundamental, se enuncia dentro de la parte social y está en relación con las condiciones de trabajo y la prevención de riesgos laborales (Martínez y Yandún, 2017). La Constitución de la República del Ecuador estipula en el artículo 326 numeral 5 que "Toda persona tendrá derecho a desarrollar sus labores en un ambiente adecuado y propicio, que garantice su salud, integridad, seguridad, higiene y bienestar"; en el numeral 6 establece que: "Toda persona rehabilitada después de un accidente de trabajo o enfermedad, tendrá derecho a ser reintegrada al trabajo y a mantener la relación laboral, de acuerdo con la ley" (Constitución República del Ecuador, 2008).

La Comunidad Andina es una organización internacional que representa varias instituciones que integran el Sistema Andino de Integración, del cual el Ecuador es miembro activo. Se han planteado como uno de los objetivos el mejorar la calidad de vida de los habitantes a través del trabajo decente por lo que impulsa la salud y seguridad como un tema estratégico que debe ser desarrollado e implementado a través de normas para minimizar accidentes laborales 
(Comunidad Andina, 2004) . La Decisión 584 "Instrumento Andino de Seguridad y Salud en el Trabajo", y su reglamento la Resolución 957 "Reglamento del Instrumento Andino de Seguridad y Salud en el Trabajo", sumado a convenios internacionales ratificados por la Organización Internacional del Trabajo, tienen como objeto promover y normar labores seguras y reducir o eliminar los daños a la salud del trabajador mediante la aplicación de medidas de prevención y control en los puestos de trabajo (Comunidad Andina, 2005).

En concordancia con el objetivo de normar un trabajo seguro en el Ecuador podemos encontrar varios cuerpos legales, entre los cuales tenemos el Decreto Ejecutivo 2393 Reglamento de Seguridad y Salud de los Trabajadores y Mejoramiento del Medio Ambiente de Trabajo, que representa una de las bases de la legislación ecuatoriana en materia de salud y seguridad, aunque su publicación data del año 1986 y se encuentra vigente, está publicado en el Registro Oficial Número 565 (Martínez y Yandún, 2017). Algunas acciones de este cuerpo legal se soportan en base a lo determinado en el Código del Trabajo ecuatoriano, en el artículo 434 manifiesta que las organizaciones que refieran más de diez trabajadores, los empleadores están obligados a obtener y presentar el reglamento interno de Higiene y Seguridad al Ministerio de Trabajo para su aprobación y será renovado cada dos años, este documento es una herramienta basal para la gestión preventiva en las organizaciones (Yandún et al., 2017).

Otro cuerpo legal a mencionar es el Acuerdo Ministerial número MDT-2017-0135, publicado en el Registro Oficial 104 de 20 de octubre de 2017, y su actualización bajo Acuerdo Ministerial número MDT-2020-001(Ministerio del Trabajo, 2020) (Ministerio del Trabajo, 2017), menciona en su Capítulo IV: "obligaciones en materia de seguridad, salud del trabajo y gestión integral de riesgos" que el empleador deberá efectuar el registro, aprobación, notificación y/o reporte de obligaciones laborales en materia de salud y seguridad en el trabajo, de forma anual en la plataforma del Sistema Único del Trabajo (SUT).

Con estos antecedentes el objetivo del presente estudio es evidenciar el cumplimiento de las directrices de la Guía de Responsabilidad Social ISO 26000 con las exigencias técnico-legales de salud y seguridad ocupacional de la legislación ecuatoriana.

\section{Metodología}

El presente estudio se basa en un estudio descriptivo no experimental de corte transversal, en el que se analiza y compara el cumplimiento del numeral 6.4.6 asunto 4 sobre prácticas laborales: salud y seguridad ocupacional de la Guía de Responsabilidad Social norma ISO 26000 (Pulido, 2018), frente a las exigencias técnicas y legales de la gestión de la salud y seguridad en el trabajo tipificadas en la legislación vigente de la República del Ecuador.

Para el cálculo de la muestra se empleó fuentes de información secundaria desde el Directorio de Empresas y Establecimientos y el Instituto Ecuatoriano de Estadísticas y Censos, tomando en cuenta como criterios de exclusión a la microempresa y pequeña empresa ya que el Ministerio de Trabajo como ente rector dispone de una exigencia mínima de requerimientos en cuanto a cumplimiento técnico legal, y también a la gran empresa quienes cumplen estándares internacionales en cuanto a Responsabilidad Social Empresarial y sistemas de gestión implementados en sus organizaciones, bajo estos parámetros la población objeto del estudio es la 
Responsabilidad Social: Cumplimiento de directrices ISO 26000 frente a los requerimientos legales de seguridad y salud en el trabajo

mediana empresa " $A$ " y mediana empresa " $B$ " con un total de 14.342 empresas registradas según el DIEE, con base a la calculadora de muestras de investigación de Question Pro se ha obteniendo una muestra $\mathrm{N}=375$ empresas con un nivel de confianza del $95 \%$ y un 5\% de margen de error, al ejecutar las encuestas se obtuvo una participación de trescientas ochenta y ocho empresas cumpliendo la muestra establecida.

En la primera fase se elaboró una encuesta que recopila información de dieciocho variables siguiendo las directrices del numeral 6.4.6.2 (Acciones y expectativas relacionadas) de la norma ISO 26000 Guía de Responsabilidad Social que tienen relación directa con el cumplimiento exigido por el Ministerio de Trabajo para empresas con más de 10 trabajadores tipificado en el acuerdo ministerial MDT-2020-001. En la segunda fase se presentó la encuesta obteniendo una participación de trescientas ochenta y ocho empresas $(\mathrm{N}=388)$, los encuestados ostentan los cargos de gerentes, responsables y jefes de Responsabilidad Social empresarial, talento humano, gestión de riesgos y seguridad y salud ocupacional. En la tercera fase del presente estudio se realizó la tabulación, análisis, discusión y recomendaciones de los datos obtenidos.

\section{Resultados}

Se ha tabulado un total de trescientas ochenta y ocho encuestas realizadas en empresas ecuatorianas, las mismas que fueron realizadas por profesionales encargados de los departamentos de Responsabilidad Social empresarial, talento humano, gestión del riesgo y seguridad y salud ocupacional, obteniendo el porcentaje más alto de participación el área de seguridad y salud ocupacional con el $45.9 \%(n=178 ; 45.9 \%)$ de las organizaciones encuestadas, como se evidencia en la Tabla 1.

\section{Tabla 1}

Áreas participantes

\begin{tabular}{lll}
\hline Áreas Participantes & & \\
\hline Variable & Frecuencia & Porcentaje \\
\hline & & \\
Responsabilidad Social Empresarial & 53 & $13.6 \%$ \\
Talento Humano & 121 & $31.2 \%$ \\
Gestión del Riesgo & 36 & $9.3 \%$ \\
Salud y Seguridad Ocupacional & 178 & $45.9 \%$ \\
& & $\mathbf{N = 3 8 8}$ \\
\hline
\end{tabular}

Fuente: Elaboración Propia.

Los resultados obtenidos del presente estudio Tabla 2, en cuanto a cumplimiento sobre prácticas laborales de salud y seguridad ocupacional de la norma ISO 26000 Guía de Responsabilidad Social que superan el 90\% se encuentran en las variables: uno 93.7\% ( $\mathrm{n}=364$; 93.7\%) ¿Dispone su organización de una política de seguridad y salud ocupacional?, cuatro 92.1\% 
$(\mathrm{n}=357 ; 92.1 \%)$ ¿La organización proporciona el equipo de protección personal y colectiva para que sus trabajadores puedan realizar sus actividades?, catorce $90.2 \%(n=350 ; 90.2 \%)$ ¿Los trabajadores pueden paralizar sus actividades ante un peligro inminente o serio que atente contra su salud y seguridad y la de otras personas?, y dieciséis 93.6\% ( $\mathrm{n}=363 ; 93.6 \%)$ ¿La empresa informa a las autoridades competentes sobre asuntos de salud y seguridad?. Los hallazgos de incumplimiento con un porcentaje superior al $44 \%$ del estudio realizado se obtienen en las siguientes variables: seis $44.1 \%(\mathrm{n}=171 ; 44.1 \%)$ ¿Se dispone de programas específicos de protección para personal vulnerable?, siete $44.8 \%(n=174 ; 44.8 \%)$ ¿Se gestiona protección equitativa en salud y seguridad a trabajadores de tiempo parcial, contratistas y subcontratistas?, quince $59.5 \%(\mathrm{n}=231 ; 59.5 \%)$ iSu empresa busca asesoramiento externo de organizaciones que tengan conocimiento del tema?, diecisiete $54.1 \%(\mathrm{n}=210 ; 54.1 \%)$ ¿Los organismos paritarios participan en decisiones y actividades relacionadas con la salud y la seguridad, incluyendo la investigación de incidentes, accidentes y enfermedades profesionales?, y dieciocho siendo la variable más representativa de incumplimiento con el 71.1\% (n=276; 71.1\%) ¿Se dispone de una política en la que se garantice que el trabajador no reciba amenazas o represalias por reportar acciones y condiciones subestándar en su actividad laboral?.

\section{Tabla 2}

Cumplimiento de directrices de la ISO 26000 en Seguridad y Salud en el Trabajo

\begin{tabular}{|c|c|c|c|c|}
\hline \multirow{2}{*}{ Nro. Pregunta } & \multicolumn{4}{|c|}{ Respuestas / Porcentajes } \\
\hline & SI (n) & $\%$ & NO (n) & $\%$ \\
\hline $\begin{array}{l}\text { 1. ¿Dispone su organización de una política de seguridad } \\
\text { y salud ocupacional? }\end{array}$ & 364 & $93.7 \%$ & 24 & $6.3 \%$ \\
\hline $\begin{array}{l}\text { 2. ¿La empresa ha implementado medidas de control de } \\
\text { los factores de riesgo críticos siguiendo el esquema } \\
\text { técnico de control en la planificación de diseño, fuente del } \\
\text { riesgo, medio de trasmisión y el trabajador? }\end{array}$ & 321 & $82.7 \%$ & 67 & $17.3 \%$ \\
\hline $\begin{array}{l}\text { 3. ¿La organización comunica a sus trabajadores las } \\
\text { prácticas y procedimientos de salud y seguridad } \\
\text { establecidos? }\end{array}$ & 262 & $67.5 \%$ & 126 & $32.5 \%$ \\
\hline $\begin{array}{l}\text { 4. ¿La organización proporciona el equipo de protección } \\
\text { personal y colectiva para que sus trabajadores puedan } \\
\text { realizar sus actividades? }\end{array}$ & 357 & $92.1 \%$ & 31 & $7.9 \%$ \\
\hline $\begin{array}{l}\text { 5. ¿La organización registra e investiga todos los } \\
\text { incidentes, accidentes y enfermedades profesionales, con } \\
\text { el objeto de minimizarlos o eliminarlos? }\end{array}$ & 289 & $74.5 \%$ & 99 & $25.5 \%$ \\
\hline $\begin{array}{l}\text { 6. ¿Se dispone de programas específicos de protección } \\
\text { para personal vulnerable? }\end{array}$ & 217 & $55.9 \%$ & 171 & $44.1 \%$ \\
\hline $\begin{array}{l}\text { 7. ¿Se gestiona protección equitativa en salud y seguridad } \\
\text { a trabajadores de tiempo parcial, contratistas y } \\
\text { subcontratistas? }\end{array}$ & 214 & $55.2 \%$ & 174 & $44.8 \%$ \\
\hline $\begin{array}{l}\text { 8. ¿Se dispone de un plan de acción para minimizar el } \\
\text { impacto de los riesgos psicosociales en el lugar de trabajo? }\end{array}$ & 242 & $62.4 \%$ & 146 & $37.6 \%$ \\
\hline
\end{tabular}


Responsabilidad Social: Cumplimiento de directrices ISO 26000 frente a los requerimientos legales de seguridad y salud en el trabajo

\begin{tabular}{|c|c|c|c|c|}
\hline \multirow{2}{*}{ Nro. Pregunta } & \multicolumn{4}{|c|}{ Respuestas / Porcentajes } \\
\hline & SI (n) & $\%$ & NO (n) & $\%$ \\
\hline $\begin{array}{l}\text { 9. ¿Se dispone de un programa de formación y } \\
\text { entrenamiento en salud y seguridad en el trabajo? }\end{array}$ & 253 & $65.2 \%$ & 135 & $34.8 \%$ \\
\hline $\begin{array}{l}\text { 10. ¿Los costos de la gestión en salud y seguridad en el } \\
\text { trabajo son asumidos en su totalidad por la organización } \\
\text { (exámenes ocupacionales, equipo de protección personal, } \\
\text { ropa de trabajo)? }\end{array}$ & 321 & $82.7 \%$ & 67 & $17.3 \%$ \\
\hline $\begin{array}{l}\text { 11. ¿Los representantes de los organismos paritarios } \\
\text { (comité, subcomités y delegados de seguridad y salud en } \\
\text { el trabajo) son elegidos de acuerdo con las directrices del } \\
\text { marco regulatorio vigente? }\end{array}$ & 337 & $86.9 \%$ & 51 & $13.1 \%$ \\
\hline $\begin{array}{l}\text { 12. ¿Los organismos paritarios disponen de la información } \\
\text { concerniente a la identificación, evaluación y medidas de } \\
\text { control de los factores de riesgo por puesto de trabajo? }\end{array}$ & 347 & $89.4 \%$ & 41 & $10.6 \%$ \\
\hline $\begin{array}{l}\text { 13. ¿Los organismos paritarios consultan y son } \\
\text { consultados, libremente, acerca de todos los aspectos de } \\
\text { salud y seguridad relacionados con su trabajo? }\end{array}$ & 295 & $76 \%$ & 93 & $24 \%$ \\
\hline $\begin{array}{l}\text { 14. ¿Los trabajadores pueden paralizar sus actividades } \\
\text { ante un peligro inminente o serio que atente contra su } \\
\text { salud y seguridad y la de otras personas? }\end{array}$ & 350 & $90.2 \%$ & 38 & $9.8 \%$ \\
\hline $\begin{array}{l}\text { 15. ¿Su empresa busca asesoramiento externo de } \\
\text { organizaciones que tengan conocimiento del tema? }\end{array}$ & 157 & $40.5 \%$ & 231 & $59.5 \%$ \\
\hline $\begin{array}{l}\text { 16. ¿La empresa informa a las autoridades competentes } \\
\text { sobre asuntos de salud y seguridad? }\end{array}$ & 363 & $93.6 \%$ & 25 & $6.4 \%$ \\
\hline $\begin{array}{l}\text { 17. ¿Los organismos paritarios participan en decisiones y } \\
\text { actividades relacionadas con la salud y la seguridad, } \\
\text { incluyendo la investigación de incidentes, accidentes y } \\
\text { enfermedades profesionales? }\end{array}$ & 178 & $45.9 \%$ & 210 & $54.1 \%$ \\
\hline $\begin{array}{l}\text { 18. ¿Se dispone de una política en la que se garantice que } \\
\text { el trabajador no reciba amenazas o represalias por reportar } \\
\text { acciones y condiciones subestándar en su actividad } \\
\text { laboral? }\end{array}$ & 112 & $28.9 \%$ & 276 & $71.1 \%$ \\
\hline
\end{tabular}

\section{Fuente: Elaboración Propia.}

\section{Discusión}

Los resultados de este estudio evidencian que hay varias manifestaciones de cumplimiento e implementación en el asunto 4 sobre prácticas laborales que indica la Guía de Responsabilidad 
Social ISO 26000 que están relacionadas con las exigencias técnico-legales de salud y seguridad ocupacional de la legislación ecuatoriana. El estudio que evalúa dieciocho prácticas de Responsabilidad Social en trescientas ochenta y ocho empresas diferentes, se evidencia las variables que superan un $80 \%$ y son las siguientes: 1 política de seguridad y salud ocupacional, 2 medidas de control de los factores de riesgo críticos, 4 proporciona el equipo de protección personal y colectiva, 10 costos de la gestión en salud y seguridad en el trabajo, 11 elección de los representantes de los organismos paritarios, 12 información concerniente a identificación, evaluación y medidas de control de los factores de riesgo, 14 paralizar sus actividades ante un peligro inminente o serio, 16 la empresa informa a las autoridades competentes sobre asuntos de salud y seguridad. En conjunto son ocho variables que forman un marco referencial que puede ser interpretado de forma positiva en temas de implementación.

En cierta medida, los resultados están relacionados con las distinciones propuestas por Martínez 2017 (Martínez y Yandún, 2017), que explican que el formular la política empresarial de seguridad y salud ocupacional está respaldada legalmente en la Decisión 584 Instrumento Andino de Seguridad y Salud en el Trabajo en su artículo 11 literal a (Comunidad Andina, 2004) y según los resultados ( $\mathrm{n}=$ obtenidos las empresas han desarrollado este compromiso empresarial.

Por otra parte las organizaciones en su mayoría han implementado los principios de la acción preventiva que refiere a la aplicación de medidas de control de los factores de riesgo siguiendo un orden técnico establecido que inicia por la planificación o diseño cuando los procesos y el sistema lo permiten, pasa por control en la fuente de riesgo, se continua en el medio de transmisión y por último se controla en el trabajador (Instituto Ecuatoriano de Seguridad Social, 2016). Con los hallazgos encontrados en el estudio, refleja que el $82.7 \%$ han cumplido los mencionados procesos en el orden técnico establecido y que los resultados evidenciados se contraponen con lo manifestado por Jorge Molado en el estudio: de la salud ocupacional a la gestión de la seguridad y salud en el trabajo (Hernando y Velandia, 2013), quien manifiesta que la implementación del control se ha dado en orden inverso, centrado inicialmente en los trabajadores haciendo énfasis en la dotación de los equipos de protección personal pasando por alto la gestión en la fuente del peligro.

En referencia a la variable que menciona la entrega de equipos de protección personal y colectiva a sus trabajadores cumple en un $92.1 \%(n=357,92.1 \%)$, Jirka Glenn y Thompson Wade (Jirka y Thompson, 2009) manifiestan que el equipo de protección personal es una prenda que utilizan los trabajadores para protegerse de los distintos factores de riesgo, estos deben ser usados cuando los diseños de ingeniería no proporcionan un entorno de trabajo seguro, siendo una obligatoriedad del empresario su dotación.

Para los organismos paritarios en el Ecuador se estipula que en todas las empresas o centros de trabajo con más de 15 trabajadores se establecerá un Comité de Seguridad e Higiene del Trabajo, el mismo que será inscrito legalmente en el Ministerio del Trabajo, debe estar integrado en forma paritaria por tres representantes de los trabajadores y tres representantes de los empleadores y sus suplentes (Ministerio del Trabajo - Ecuador, 1986.). Los resultados del estudio reflejan que los representantes de los organismos paritarios son elegidos de acuerdo con las directrices del marco regulatorio vigente en el Ecuador con el 86.9\% ( $\mathrm{n}=337,86.9 \%)$. En la variable que determina si los trabajadores son consultados, libremente, acerca de todos los aspectos 
Responsabilidad Social: Cumplimiento de directrices ISO 26000 frente a los requerimientos legales de seguridad y salud en el trabajo

de salud y seguridad relacionados con su trabajo el resultado es $76 \%(\mathrm{n}=295,76 \%)$, la variable que pregunta si disponen de la información concerniente a la identificación, evaluación y medidas de control de los factores de riesgo por puesto de trabajo obtuvo el resultado de $89.4 \%$ ( $n=347$, 89.4\%). Los resultados descritos se encuentran en concordancia con lo expuesto por Josefa Romeral quien manifiesta en su publicación denominada Gestión de la seguridad y salud laboral, y mejora de las condiciones de trabajo (Romeral Hernández, 2012) manifestando que los organismos paritarios son importantes en la gestión preventiva y sus funciones vitales entre otras son desarrollar programas de prevención de riesgos, implementar proyectos en materia de prevención psicosocial y otros factores de riesgo, mejorar la organización del trabajo, comunicar e informar toda información que esté relacionado con materia preventiva.

Se observa debilidades en la consecución de las directrices emitidas por la Guía de Responsabilidad Social en el contexto del compromiso, protección y equidad de todos los grupos de interés de las organizaciones. La gestión de la salud y seguridad en el trabajo conjuntamente con las buenas prácticas emitidas por la ISO 26000 tienen una estrecha relación en su objetivo fundamental de protección a los trabajadores, con los datos reflejados en el estudio, la variable 6 se dispone de programas específicos de protección para personal vulnerables y 7 se gestiona protección equitativa en salud y seguridad a trabajadores de tiempo parcial, contratistas y subcontratistas, evidencian un porcentaje superior al $44 \%$ de incumplimiento, la República del Ecuador tiene ratificados varios convenios con la Organización Internacional del Trabajo entre ellos el convenio 111 (Ministerio del Trabajo, 2016) sobre la discriminación (empleo y ocupación) que manifiesta: "llevar a cabo una política nacional que promueva, por métodos adecuados a las condiciones y a las prácticas nacionales, la igualdad de oportunidades y de trato en materia de empleo y ocupación, con objeto de eliminar cualquier discriminación a este respecto", con esta premisa las organizaciones deben reformular sus procedimientos de inclusión a personas vulnerables y grupos de interés. Otro de los hallazgos relevantes encontrados en un 54.1\% ( $\mathrm{n}=210$, $54.1 \%$ ) de incumplimiento es la falta de compromiso de los organismos paritarios en su participación en decisiones y actividades relacionadas con la salud y la seguridad, incluyendo la investigación de incidentes, accidentes y enfermedades profesionales, ante lo cual los responsables de prevención de riesgos laborales deberían realizar una supervisión más efectiva y proactiva de los mencionados comités. A pesar de encontrarse tipificado en el código de trabajo del Ecuador acerca de la protección de los trabajadores de no recibir represalias por parte de las empresas, se evidencia en un porcentaje alto $71.1 \%(n=276,71.1 \%)$, que no se garantiza a través de una política de protección para los trabajadores sobre denuncias de acciones y condiciones subestándar ante los entes de control por lo cual pueden estar expuestos a represalias e incluso despidos de sus puestos de trabajo.

El presente estudio refleja el resultado de las dieciocho variables tabuladas, las mismas que podrían proyectar una tendencia al cambio si las organizaciones fuesen conscientes del objetivo de la Responsabilidad Social Empresarial que es la sostenibilidad en base al equilibrio de lo social, ambiental y económico y no solo en cumplir la legislación vigente. Los autores dejamos las puertas abiertas para próximas investigaciones acerca del tema y la relación directa entre la Responsabilidad Social empresarial y el cumplimiento técnico legal en salud y seguridad laboral en el Ecuador. 


\section{Conclusiones}

La Guía de Responsabilidad Social ISO 26000 emite directrices de carácter voluntario para las organizaciones basadas en estándares exigentes para lograr que sean sostenibles y sustentables en el vertiginoso mundo globalizado en que nos encontramos, los resultados obtenidos en el presente estudio de las variables analizadas de las prácticas laborales de salud y seguridad ocupacional de la norma ISO 26000 Guía de Responsabilidad Social evidencian que la gestión no solo debe ser documental en su cumplimiento legal de acuerdo a las exigencias del Ministerio de Trabajo del Ecuador concordando por lo descrito por (Suasnavas et al., 2019) en el estudio Responsabilidad social y gestión de la seguridad y salud en el trabajo: panorama actual de las empresas ecuatorianas, ya que esto no garantiza la pertinencia de las partes interesadas con y hacia la organización, antes de pensar en un proceso de responsabilidad social empresarial, se debe socializar de forma transversal con todos los actores el compromiso de lograr los Objetivos de Desarrollo Sostenible tres y ocho que hacen referencia a garantizar una vida sana promoviendo el bienestar en todas las edades y el crear empleos decentes para mejorar los estándares de vida, este criterio va en relación con el estudio Credibilidad en Latinoamérica del informe de Responsabilidad Social Corporativa planteado por (Sierra-García et al., 2014). Los autores dejamos las puertas abiertas para los responsables de los departamentos de talento humano, salud $\mathrm{y}$ seguridad ocupacional y responsabilidad social empresarial para incentivar futuras investigaciones acerca del tema y la relación directa entre la responsabilidad social empresarial y el cumplimiento técnico legal en salud y seguridad laboral en el Ecuador.

\section{Referencias Bibliográficas}

Castka, P., \& Balzarova, M. A. (2008). ISO 26000 and supply chains-On the diffusion of the social responsibility standard. International Journal of Production Economics, 111(2), 274-286. https://doi.org/10.1016/j.ijpe.2006.10.017

Céspedes Socarrás, G. M., y Martínez Cumbrera, J. M. (2016). Un análisis de la seguridad y salud en el trabajo en el sistema empresarial cubano. In Revista Latinoamericana de Derecho Social 22(22), 3-61. Universidad Nacional Autonoma de Mexico. https://doi.org/10.1016/j.rlds.2016.03.001

Chandan, H. C., \& Das, R. (2017). Evolution of Responsible and Sustainable Corporate Identity for Chinese Firms. In The China Business Model: Originality and Limits. Elsevier Ltd. https://doi.org/10.1016/B978-0-08-100750-1.00004-8

Comunidad Andina. (2004). Instrumento Andino de Seguridad y Salud en el Trabajo. Instrumento Andino de Seguridad y Salud En El Trabajo, 13.

Comunidad Andina. (2005). Resolución 957. Reglamento del Instrumento Andino. 6.

Constitución República del Ecuador. (2008). Derechos del buen vivir. Constitución Del Ecuador, 132.

Creixans-Tenas, J., Coenders, G., \& Arimany-Serrat, N. (2019). Corporate social responsibility and financial profile of Spanish private hospitals. Heliyon, 5(10), e02623. https://doi.org/10.1016/J.HELIYON.2019.E02623

Escamilla Solano, S., Plaza Casado, P., \& Flores Ureba, S. (2016). Analysis of the disclosure of information on corporate social responsibility in urban public transport companies in Spain. Revista de Contabilidad-Spanish Accounting Review, 19(2), 195-203. 
Responsabilidad Social: Cumplimiento de directrices ISO 26000 frente a los requerimientos legales de seguridad y salud en el trabajo

https://doi.org/10.1016/j.rcsar.2015.05.002

Gallardo-Vázquez, D., Sánchez-Hernández, M. I., \& Corchuelo-Martínez-Azúa, M. B. (2013). Validation of a measurement scale for the relationship between the orientation to corporate social responsibility and other business strategic variables. Revista de ContabilidadSpanish Accounting Review, 16(1), 11-23. https://doi.org/10.1016/S1138-4891(13)700025

Hernando, J., y Velandia, M. (2013). De la salud ocupacional a la gestión de la seguridad y salud en el trabajo: más que semántica, una transformación del sistema general de riesgos laborales. 21-32.

Instituto Ecuatoriano de Seguridad Social. (2016). Reglamento del seguro general de riesgos del trabajo (Resolución C.D. 513). Iess, 53(9), 1689-1699. https://www.iess.gob.ec/documents/10162/33703/C.D.+513

Jirka, G. P., \& Thompson, W. (2009). Personal protective equipment. The A3 Workbook: Unlock Your Problem-Solving Mind, 493-508. https://doi.org/10.1201/9781420071825-29

Larrosa Moles, M., \& Martínez-Jarreta, B. (2020). Occupational cancer: A hidden reality and an awaiting challenge. Medicina Clinica, 154(1), 23-28. https://doi.org/10.1016/j.medcli.2019.08.005

Liu, R., Liu, Z., Liu, H. C., \& Shi, H. (2021). An improved alternative queuing method for occupational health and safety risk assessment and its application to construction excavation. Automation in Construction, 126, 103672. https://doi.org/10.1016/j.autcon.2021.103672

López Gobernado, M., Villalba Gil, D., \& Hernández Bartolomé, J. (2018). Health and safety management system to improve the self-protection plans in intensive care units. Medicina Intensiva, 42(7), 457. https://doi.org/10.1016/j.medin.2018.02.010

López Salazar, A., Ojeda Hidalgo, J. F., \& Ríos Manríquez, M. (2017). The corporate social responsibility from the perception of human capital. A case study. Revista de ContabilidadSpanish Accounting Review, 20(1), 36-46. https://doi.org/10.1016/j.rcsar.2016.01.001

Martinez Ferrero, J., Prado Lorenzo, J. M., \& Fernandez-Fernandez, J. M. (2013). Corporate social responsibility vs. accounting ethics. Revista de Contabilidad-Spanish Accounting Review, 16(1), 32-45. https://doi.org/10.1016/S1138-4891(13)70004-9

Martínez, M., y Yandún, E. D. (2017). Seguridad y Salud Ocupacional en Ecuador: Contribución Normativa a la Responsabilidad Social Organizacional. INNOVA Research Journal, 2(3), 58-68. https://doi.org/10.33890/innova.v2.n3.2017.135

Ministerio del Trabajo. (2016). Código del Trabajo. Registro Oficial, 138, 1-159. www.lexis.com.ec

Ministerio del Trabajo. (2017). Instructivo para el cumplimiento de las obligaciones de empleadores. Acuerdo Ministerial 135, 1-12. www.lexis.com.ec

Ministerio del Trabajo. (2020). ACUERDO-MINISTERIAL-Nro.-MDT-2020-001.pdf.

Ministerio del Trabajo - Ecuador. (n.d.). Decreto ejecutivo 2393 Reglamento de seguridad y salud de los trabajadores y mejoramiento del medio ambiente de trabajo.

Moreno, G., y Villena, R. (2020). Vacunas en salud laboral... una oportunidad para prevenir e invertir en salud. Revista Médica Clínica Las Condes, 31(3), 287-303. https://doi.org/10.1016/j.rmclc.2020.05.001

Olanipekun, A. O., Omotayo, T., \& Saka, N. (2021). Review of the Use of Corporate Social Responsibility (CSR) Tools. Sustainable Production and Consumption, 27, 425-435. https://doi.org/10.1016/J.SPC.2020.11.012

Esta obra se comparte bajo la licencia Creative Common Atribución-No Comercial 4.0 International (CC BY-NC 4.0) 
Pishvaee, M. S., Mohseni, S., \& Bairamzadeh, S. (2021). Sustainability concepts in biofuel supply chain. Biomass to Biofuel Supply Chain Design and Planning Under Uncertainty, 95-126. https://doi.org/10.1016/B978-0-12-820640-9.00005-2

Pulido, M. P. (2018). ISO 26000:2010 Guidance on Social Responsibility. In Ethics Management in Libraries and Other Information Services. https://doi.org/10.1016/b978-0-08-101894$1.00005-7$

Rajendran, S., Giridhar, S., Chaudhari, S., \& Gupta, P. K. (2021). Technological advancements in occupational health and safety. Measurement: Sensors, 15, 100045. https://doi.org/10.1016/j.measen.2021.100045

Romeral Hernández, J. (2012). Gestión de la seguridad y salud laboral, y mejora de las condiciones de trabajo. El modelo español. In Boletín Mexicano de Derecho Comparado, nueva serie, año XLV, núm. 135, septiembre-diciembre de 2012, pp. 1325-1339.Biblioteca Jurídica Instituto de Investigaciones Jurídicas de la UNAM (Issue siglo I, pp. 1325-1339).

Romero Saldaña, M., Moreno Pimentel, A. G., \& Santos Posada, A. (2019). Occupational Health Nursing: Competence and experience to achieve the safety, health and well-being of the working population. Enfermeria Clinica, 29(6), 376-380. https://doi.org/10.1016/j.enfcli.2019.09.012

Shanmugasundar, G., Sri Sabarinath, S., Ramesh Babu, K., \& Srividhya, M. (2021). Analysis of occupational health and safety measures of employee in material manufacturing industry using statistical methods. Materials Today: Proceedings. https://doi.org/10.1016/j.matpr.2020.11.296

Sharma, R., \& Mishra, D. K. (2021). An analysis of thematic structure of research trends in occupational health and safety concerning safety culture and environmental management. $\begin{array}{lllll}\text { Journal of } & \text { Cleaner } & \text { Production, } & 281, & \end{array}$ https://doi.org/10.1016/j.jclepro.2020.125346

Sierra-García, L., García-Benau, M. A., \& Zorio, A. (2014). Credibility in Latin America of corporate social responsibility reports. RAE Revista de Administracao de Empresas, 54(1), 28-38. https://doi.org/10.1590/S0034-759020140104

Suasnavas, P., Andrade, A., Granda, K., Dávalos, H., Cárdenas, H., y Gómez, A. (2019). Responsabilidad social y gestión de la seguridad y salud en el trabajo: panorama actual de las empresas ecuatorianas. Revista ESPACIOS, 40(04), 18. http://es.revistaespacios.com/a19v40n04/19400418.html

Yandún, E., Freire, L., Flores, J. C., y Gualotuña, C. A. V. (2017). Responsabilidad social corporativa y su relación con la seguridad y salud ocupacional en el Ecuador. RECIMUNDO: Revista Científica de la Investigación y el Conocimiento, 1(5), 766-798. 